\title{
RANDOM FIXED POINTS AND RANDOM APPROXIMATIONS IN NONCONVEX DOMAINS
}

\author{
ABDUL RAHIM KHAN and A. B. THAHEEM \\ King Fahd University of Petroleum and Minerals \\ Department of Mathematical Sciences \\ Dhahran 31261, Saudi Arabia \\ NAWAB HUSSAIN \\ Bahauddin Zakariya University \\ Centre for Advanced Studies in Pure and Applied Mathematics \\ Multan 60800, Pakistan
}

(Received November, 2000; Revised June, 2001)

\begin{abstract}
Stochastic generalizations of some fixed point theorems on a class of nonconvex sets in a locally bounded topological vector space are established. As applications, Brosowski-Meinardus type theorems about random invariant approximation are obtained. This work extends or provides stochastic versions of several well known results.

Key words: Random Fixed Point, Random Approximation, Nonexpansive Random Map, Banach Operator, Contractive Map, Random Operator, Locally Bounded Topological Vector Space.
\end{abstract}

AMS subject classifications: 41A65, 46A16, 47H10, 60H25.

\section{Introduction}

Probabilistic functional analysis is an important mathematical discipline because of its applications to probabilistic models in applications. Random operator theory is needed for the study of various classes of random equations. The study of random fixed point theorems was initiated by the Prague school of probabilists in the 1950s. Random fixed point theorems and random approximations are stochastic generalizations of classical fixed point and approximation theorems, and find their applications in probability theory and nonlinear analysis. The random fixed point theory for self-maps and nonself-maps has been developed by various authors (see e.g., [2-3, 13, 19]). Recently, this theory has been further extended for 1-set contractive mappings that include condensing, nonexpansive, semicontractive and completely continuous random maps, etc.

Brosowski [4] initiated the study of invariant approximations using the fixed point theory and subsequently various generalizations of Brosowski's results have appeared in the literature (see for example, [7, 12]).

In this paper, we prove some random fixed point theorems for nonexpansive random operators defined on a class of nonconvex sets containing the subclass of starshaped sets in a locally bounded topological vector space. As applications of our results, we obtain Brosowski-Meinardus type results on random invariant approximation (cf. [4, 14]). These results are obtained in Section 3, while in Section 2 we recall certain technical deinitions and known results. 


\section{Preliminaries}

A subset $S$ of a (real or complex) linear space $X$ is called starshaped if there exists at least one point $z \in S$ such that $t z+(1-t) x \in S$ for all $x \in S, t \in(0,1)$; $z$ is called a star center of $S$. Let $0<p \leq 1$. A real-valued map $\|\cdot\|_{p}$ on a linear space $X$ is called $p$-norm if

(i) $\quad\|x\|_{p} \geq 0$ and $\|x\|_{p}=0$ iff $x=0$,

(ii) $\quad\|\lambda x\|_{p}=|\lambda|^{p}\|x\|_{p}$, and

(iii) $\quad\|x+y\|_{p} \leq\|x\|_{p}+\|y\|_{p}$ for all $x, y \in E$ and $\lambda \in \mathbb{C}$.

The formula $d_{p}(x, y)=\|x-y\|_{p}$ defines a translation invariant metric on $X$. It is well known that the topology of any Hausdorff locally bounded topological vector space is given by some $p$-norm. In the sequel, unless mentioned specifically otherwise, $X$ denotes a Hausdorff separable complete locally bounded topological vector space (not necessarily locally convex) whose topology is generated by a $p$-norm. Of course, if $p=1$, then $X$ will stand for a separable Banach space.

Let $S \subseteq X$ and $\mathcal{F}=\left\{f_{\alpha}\right\}_{\alpha \in S}$ a family of functions from $[0,1]$ into $S$ such that $f_{\alpha}(1)=\alpha$ for each $\alpha \in S$. Following Dotson [6], the family $\mathcal{F}$ is said to be contractive if there exists a function $\phi:(0,1) \rightarrow(0,1)$ such that for all $\alpha, \beta \in S$ and all $t \in[0,1]$, we have $\left\|f_{\alpha}(t)-f_{\beta}(t)\right\|_{p} \leq[\phi(t)]^{p}\|\alpha-\beta\|_{p}$. The family $\mathcal{F}$ is said to be jointly continuous (resp. jointly weakly continuous) if $t \rightarrow t_{0}$ in $[0,1]$ and $\alpha \rightarrow \alpha_{0}$ in $S$ (resp. $t \rightarrow t_{0}$ in $[0,1]$ and $\alpha \stackrel{w}{\rightarrow} \alpha_{0}$ in $S$ ), then $f_{\alpha}(t) \rightarrow f_{\alpha_{0}}\left(t_{0}\right)$ (resp. $\left.f_{\alpha}(t) \stackrel{w}{\rightarrow} f_{\alpha_{0}}(t)\right)$ in $S$ ( $w$ denotes the weak convergence).

We observe that if $S \subseteq X$ is starshaped with $z$ as star center and $f_{x}(t)=(1-t) z+t x$, $(x \in S ; t \in[0,1])$, then $\mathcal{F}=\left\{f_{\alpha}\right\}_{\alpha \in S}$ is a contractive jointly continuous family with $\phi(t)=t$. Thus the class of subsets of $X$ with the property of contractiveness and joint continuity contains the class of starshaped sets which in turn contains the class of convex sets.

Throughout this paper, $(\Omega, \mathcal{A})$ denotes a measurable space if not mentioned otherwise. A mapping $T: \Omega \rightarrow 2^{X}$ is said to be measurable if for any open subset $C$ of $X, T^{-1}(C)=\{\omega \in \Omega: T(\omega) \cap C \neq \emptyset\} \in \mathcal{A}$. Let $S$ be a nonempty subset of $X$. A mapping $f: \Omega \times S \rightarrow X$ is called a random operator if for any $x \in S, f(\cdot, x)$ is measurable. A measurable mapping $\xi: \Omega \rightarrow S$ is called a random fixed point of a random operator $f: \Omega \times S \rightarrow X$ if for every $\omega \in \Omega, \xi(\omega)=f(\omega, \xi(\omega))$.

Suppose that $\mathcal{H}=\left\{f_{\alpha}\right\}_{\alpha \in S}$ is a family of functions from $[0,1]$ into $S$ having the property that for each sequence $\left(\lambda_{n}\right)$ in $(0,1]$, with $\lambda_{n} \rightarrow 1$ as $n \rightarrow \infty$, we have

$$
f_{x}\left(\lambda_{n}\right)=\lambda_{n} x
$$

We observe that $\mathcal{H} \subseteq \mathcal{F}$ and it has the additional property that it is contractive, jointly continuous and jointly weakly continuous.

If for a subset $S$ of $X$, there exists a contractive jointly continuous family of functions $\mathcal{F}=\left\{f_{\alpha}\right\}_{\alpha \in S}$ (resp. a family of functions satisfying $\left({ }^{*}\right)$ ), then we say that $S$ has the property of contractiveness and joint continuity (resp. property $(*)$ ).

Example 2.1: Any subspace, a convex set with 0, a starshaped subset with center 0 and a cone of a $p$-normed space have the family of functions associated with them which satisfy condition (*).

Let $T: \Omega \times S \rightarrow S$ be a random operator. If we restrict it to the family $\mathcal{H}$, then the operators $T_{n}$ defined by $T_{n}(\omega, x)=f_{T(\omega, x)}\left(\lambda_{n}\right)=\lambda_{n} T(\omega, x)$ are random operators because of the randomness of $T$.

A map $f: S \rightarrow X$ is called

(i) nonexpansive if $\|f(x)-f(y)\|_{p} \leq\|x-y\|_{p}$ for all $x, y \in S$

(ii) Banach operator if there is a constant $k, 0 \leq k<1$ such that $\left\|f(x)-f^{2}(x)\right\|_{p} \leq k\|x-f(x)\|_{p}$, for all $x \in S$

(iii) isometric if $d_{p}(f(x), f(y))=d_{p}(x, y)$ for all $x, y \in S$. 
A random operator $f: \Omega \times S \rightarrow X$ is continuous (nonexpansive, Banach operator, etc.) if for each $\omega \in \Omega, f(\omega, \cdot)$ is continuous (nonexpansive, Banch operator, etc.). For any $x \in X$, we set $d_{p}(x, S)=\inf \left\{\|x-y\|_{p}: y \in S\right\}$ and $P_{S}(x)=\left\{y \in S: \quad\|x-y\|_{p}=d_{p}(x, S)\right\}$; $P_{S}(x)$ is called the set of all best approximations of $x$ from $S$ in $X$.

A subspace $M$ of $X$ is said to be

(i) pseudo-Chebyshev if for each $x \in X, P_{M}(x)$ is a nonempty and finite dimensional set in $X$

(ii) quasi-Chebyshev if for each $x \in X, P_{M}(x)$ is a nonempty and compact set in $X$.

Every finite dimensional subspace of a Banach space $X$ is pseudo-Chebyshev. Moreover, every pseudo-Chebyshev subspace is quasi-Chebyshev but not conversely, in general (see [15] for more details).

We say that $X^{*}$ (the dual of $X$ ) is separating if for each nonzero $x \in X$, there exists $\phi \in X^{*}$ such that $\phi(x) \neq 0$. In this case, the weak topology of $X$ is Hausdorff ([11]).

We shall need the following results.

Theorem A: ([11, Theorem 3.1]). Let $S$ be a compact subset of $X$. If $\mathcal{F}$ is a family of contractive and jointly continuous functions associated with $S$, then any nonexpansive selfmap $T$ of $S$ has a fixed point.

Theorem B: ([11, Theorem 3.4]). Let $S$ be a weakly compact subset of $X$ with separating dual $X^{*}$. Suppose that $S$ has the property of contractiveness and joint continuity. Then any nonexpansive selfmap $T$ of $S$ has a fixed point.

\section{Main Results}

We begin with the following "random fixed point theorem" for nonexpansive operators which extends Theorem 1 of Dotson [6].

Theorem 3.1: Suppose that $S$ is a compact subset of $X$ with the property $(*)$ and $T: \Omega \times S \rightarrow S$ is a nonexpansive random operator. Then $T$ has a random fixed point.

Proof: By assumption, there is a family $\mathcal{H}=\left\{f_{\alpha}\right\}_{\alpha \in S}$ satisfying $(*)$. If we take $\lambda_{n}=\frac{n}{n+1} \in(0,1]$ and define $T_{n}: \Omega \times S \rightarrow S$ by $T_{n}(\omega, x)=f_{T(\omega, x)}\left(\lambda_{n}\right), n=1,2, \ldots$, the continuity of $T$ and its randomness imply that each $T_{n}$ is a continuous random operator from $S$ into itself. We first show that each $T_{n}$ is a Banach operator.

$$
\begin{aligned}
\left\|T_{n}(\omega, x)-T_{n}^{2}(\omega, x)\right\|_{p} & \left.=\| f_{T(\omega, x)}\left(\lambda_{n}\right)-f_{T\left(\omega, f_{T(\omega, x)}\left(\lambda_{n}\right)\right.}\right)\left(\lambda_{n}\right) \|_{p} \\
& \leq\left[\phi\left(\lambda_{n}\right)^{p}\left\|T(\omega, x)-T\left(\omega, f_{T(\omega, x)}\left(\lambda_{n}\right)\right)\right\|_{p}\right. \\
& \leq\left[\phi\left(\lambda_{n}\right)\right]^{p}\left\|x-f_{T(\omega, x)}\left(\lambda_{n}\right)\right\|_{p} \\
& \left.=\left[\phi\left(\lambda_{n}\right)\right]^{p} \| x-T_{n}(\omega, x)\right) \|_{p}
\end{aligned}
$$

for each $\omega \in \Omega$.

By [3, Theorem 2.1], $T_{n}$ has a random fixed point $\xi_{n}$. For each $n$, define $G_{n}: \Omega \rightarrow K(S)$ by $G_{n}(\omega)=\overline{\left\{\xi_{i}(\omega): i \geq n\right\}}$ where $K(S)$ is the set of all nonempty compact subsets of $S$. Define $G: \Omega \rightarrow K(S)$ by $G(\omega)=\bigcap_{n=1}^{\infty} G_{n}(\omega)$. Then $G$ is measurable by [8, Theorem 4.1] and hence it has a measurable selector $\xi$. We now show that $\xi$ is the random fixed point of $T$. Fix $\omega \in \Omega$. Since $\xi(\omega) \in G(\omega)$, therefore there exists a sub sequence $\left(\xi_{n_{j}}(\omega)\right)$ of $\left(\xi_{n}(\omega)\right)$ that converges to $\xi(\omega)$. Since $T_{n_{j}}\left(\omega, \xi_{n_{j}}(x)\right)=\xi_{n_{j}}(\omega)$, we have $T_{n}\left(\omega, \xi_{n_{j}}(\Omega)\right) \rightarrow \xi(\omega)$. The continuity of $T(\omega, \cdot)$ implies $T\left(\omega, \xi_{n_{j}}(\omega)\right) \rightarrow T(\omega, \xi(\omega))$ and hence, using $\lambda_{n_{j}} \rightarrow 1$ and the joint continuity, we get $T_{n_{j}}\left(\omega, \xi_{n_{j}}(\omega)\right)=$ $f_{T\left(\omega, \xi_{n_{j}}(\omega)\right)}\left(\lambda_{n_{j}}\right) \rightarrow f_{T(\omega, \xi(\omega))}(1)=T(\omega, \xi(\omega))$. As $X$ is Hausdorff, we get 
$T(\omega, \xi(\omega))=\xi(\omega)$.

As an immediate consequence of the above theorem, we have the following BrosowksiMeinardus type theorem on best random approximation and random fixed points.

Theorem 3.2: Suppose that $T: \Omega \times X \rightarrow X$ is a nonexpansive random operator with deterministic fixed point $x$. Assume further that $T$ leaves a compact subset $M$ of $X$ as invariant and $P_{M}(x)$ has the property $(*)$. Then $x$ has a best random approximation $\xi: \Omega \rightarrow M$ which is also a random fixed point of $T$.

Proof: It is easy to see that $P_{M}(x)$ is nonempty. Let $b \in P_{M}(x)$. Then for each $\omega \in \Omega$, $T(\omega, x)=x$. As $T(\omega, \cdot) \quad$ is nonexpansive, so $\quad d_{p}(x, M) \leq\|x-T(\omega, b)\|_{p}=$ $\|T(\omega, x)-T(\omega, b)\|_{p} \leq\|x-b\|_{p}=d_{p}(x, M)$. So $\|x-T(\omega, b)\|_{p}=d_{p}(x, M)$ for each $b \in P_{M}(x)$. Therefore, $P_{M}(x)$ is $T(\omega, \cdot)$-invariant for each $\omega \in \Omega$. Also, $P_{M}(x)$ being a closed subset of a compact subset is compact. Therefore, by Theorem 3.1, $T$ has a random fixed point in $P_{M}(x)$.

The following corollary provides a random generalized version of Theorem 3 in [16] and extends Theorem 4 in [2].

Corollary 3.3: Let $X$ be a separable Banach space. Suppose that $T: \Omega \times X \rightarrow X$ is a nonexpansive random operator with deterministic fixed point $x$. Assume that $T(\omega, \cdot)$ leaves a quasi-Chebyshev subspace $M$ invariant. Then the point $x$ has a random best approximation $\xi: \Omega \rightarrow M$ which is also a random fixed point of $T$.

Proof: The set $P_{M}(x)$ is nonempty and compact for each $x$ in $X$. As in the proof of Theorem 3.2, $P_{M}(x)$ is $T(\omega, \cdot)$-invariant for each $\omega \in \Omega$. Hence the result follows from Theorem 3.1.

Note that Theorem 2.1 of Beg and Shahzad [3] remains true for a closed subset $S$ of a separable metric space $X$ and $\overline{T(\omega, S)}$ is compact for each $\omega \in \Omega$ (cf. Remark 2.2 [3]). Consequently we obtain the following version of Theorem 3.1 which gives an extension of Theorem 3 of Beg and Shahzad [2].

Theorem 3.4: Let $S$ be a closed subset of $X$ and $T: \Omega \times S \rightarrow S$ a nonexpansive random operator. Assume that $\overline{T(\omega, S)}$ is a compact set for each $\omega \in \Omega$. If $S$ has a family $\mathcal{H}$ with the property $(*)$, then $T$ has a random fixed point.

The following is a random analog of Theorem 2 of Dotson [6].

Theorem 3.5: Let $X$ be a separable Banach space and $S$ a weakly compact subset of $X$. Suppose that $S$ has a family $\mathcal{H}$ with the property $(*)$ and $T: \Omega \times S \rightarrow S$ is a weakly continuous nonexpansive random operator. Then $T$ has a random fixed point.

Proof: As in the proof of Theorem 3.1, let $\lambda_{n}=\frac{n}{n+1}, n=1,2, \ldots$. Define mappings $T_{n}: \Omega \times S \rightarrow S$ by $T_{n}(\omega, x)=f_{T(\omega, x)}\left(\lambda_{n}\right)$. Then for any $\omega \in \Omega$ and $x, y \in S$, we have

$$
\begin{aligned}
\left\|T_{n}(\omega, x)-T_{n}(\omega, y)\right\| & =\left\|f_{T(\omega, x)}\left(\lambda_{n}\right)-f_{T(\omega, y)}\left(\lambda_{n}\right)\right\| \\
& \leq \phi\left(\lambda_{n}\right)\|T(\omega, x)-T(\omega, y)\| \\
& \leq \phi\left(\lambda_{n}\right)\|x-y\| .
\end{aligned}
$$

Hence, each $T_{n}$ has a random fixed point $\xi_{n}$ (see [9]) and so $T_{n}\left(\omega, \xi_{n}(\omega)\right)=\xi_{n}(\omega)$ for each $\omega \in \Omega$. Now for each $n$, define $G_{n}: \Omega \rightarrow W K(S)$ by $G_{n}(\omega)=w$-cl $\left\{\xi_{i}(\omega): n \leq i\right\}$, where $\omega-\mathrm{cl}(A)$ denotes the weak closure of $A$ and $W K(S)$ is the set of all nonempty weakly compact subsets of $S$. Define $G: \Omega \rightarrow W K(S)$ by $G(\omega)=\bigcap_{n=1}^{\infty} G_{n}(\omega)$. Then arguments similar to those in [5, Theorem 6.3.2] ensure the existence of a measurable selector $\xi(\omega)$ of $G(\omega)$. We show that $\xi$ is a random fixed point of $T$. Fix

$\omega \in \Omega$. Since $\xi(\omega) \in G(\omega)$, therefore there exists a subsequence $\left(\xi_{n_{j}}(\omega)\right)$ of $\left(\xi_{n}(\omega)\right)$ that converges weakly to $\xi(\omega)$; that is, $\xi_{n_{j}}(\omega) \stackrel{w}{\rightarrow} \xi(\omega)$. Since $T_{n_{j}}\left(\omega, \xi_{n_{j}}(\omega)\right)=\xi_{n_{j}}(\omega)$, we have $T_{n_{j}}\left(\omega, \xi_{n_{j}}(\omega)\right) \stackrel{w}{\rightarrow} \xi(\omega)$. The weak continuity of $T(\omega, \cdot)$ implies 


$$
T\left(\omega, \xi_{n_{j}}(\omega)\right) \stackrel{w}{\rightarrow} T(\omega, \xi(\omega))
$$

and hence, using the joint weak continuity, we get

$$
T_{n_{j}}\left(\omega, \xi_{n_{j}}(\omega)\right)=f_{T\left(\omega, \xi_{n_{j}}(\omega)\right)}\left(\lambda_{n_{j}}\right) \stackrel{w}{\rightarrow} f_{T(\omega, \xi(\omega))}(1)=T(\omega, \xi(\omega)) .
$$

By the Hausdorff property of the weak topology, we get the required result $T(\omega, \xi(\omega))=\xi(\omega)$.

The following result generalizes Theorem 8 of Habinaik [7].

Theorem 3.6: Let $T: \Omega \times X \rightarrow X$ be a weakly continuous nonexpansive random operator with deterministic fixed point $x$. Assume that $T$ leaves a weakly compact subset $M$ of $X$ invariant, $T(\omega, \cdot) \mid M$ is compact and $P_{M}(x)$ has the property $(*)$. Then the point $x$ has a best random approximation $\xi: \Omega \rightarrow M$ which is also a random fixed point of $T$.

Proof: The proof is analogous to that of Theorem 3.2 and is therefore omitted.

An affine continuous map is weakly continuous so we deduce from Theorem 3.5, the following random version of Corollary 1.10 of Veeramani [18].

Corollary 3.7: Let $S$ be a weakly compact convex subset of a separable Banach space $X$. If $T: \Omega \times S \rightarrow S$ is an affine nonexpansive random operator, then $T$ has a random fixed point.

Corollary 3.8: Let $S$ be a weakly compact convex subset of a strictly convex separable Banach space $X$. If $T: \Omega \times S \rightarrow S$ is an isometric random operator, then $T$ has a random fixed point.

Proof: It is sufficient to show that $T(\omega, \cdot)$ is affine for each $\omega \in \Omega$. Let $x, y \in S$ and $z=t x+(1-t) y$, for any $t$ in $[0,1]$. Then we have

$$
\|T(\omega, x)-T(\omega, z)\|=\|x-z\|=(1-t)\|x-y\|=(1-t)\|T(\omega, x)-T(\omega, y)\|
$$

and

$$
\|T(\omega, y)-T(\omega, z)\|=\|y-z\|=t\|x-y\|=t\|T(\omega, x)-T(\omega, y)\| .
$$

By the strict convexity of $X$, we get $T(\omega, z)=t T(\omega, x)+(1-t) T(\omega, y)$, for each $t$ in $[0,1]$ and $\omega \in \Omega$.

An application of Corollary 3.7 leads to the following random analog of a result of Brosowski [4].

Corollary 3.9: Let $X$ be a separable Banach space and $T: \Omega \times X \rightarrow X$ a linear nonexpansive random operator with deterministic fixed point $x$. Let $M$ be a $T(\omega, \cdot)$-invariant subset of $X$. If $P_{M}(x)$ is nonempty convex and compact, then the point $x$ has a random best approximation $\xi: \Omega \rightarrow M$ which is also a random fixed point of $T$.

Proof: As before, we can show that $P_{M}(x)$ is $T(\omega, \cdot)$-invariant and so the conclusion follows from Corollary 3.7 .

In the presence of a measure $\mu$ on $(\Omega, \mathcal{A})$, we can talk about a zero set. This is the case, if for example, $\mu$ is a $\sigma$-finite measure, then a subset $N$ of $\Omega$ is a zero set provided $\mu(N)=0$. In this case, a measurable map $\xi: \Omega \rightarrow S$ is said to be a random fixed point of a random operator $f: \Omega \times S \rightarrow X$ if $\xi(\omega)=f(\omega, \xi(\omega))$ for almost all $\omega \in \Omega$. That is, there may exist a zero set $N$ with $\mu(N)=0$ such that $\xi(\omega)=f(\omega, \xi(\omega))$ for all $\omega \in \Omega \backslash N$ (the difference set of $\Omega$ and $N)$.

The Borel $\sigma$-algebra $B(X)$ is the smallest $\sigma$-algebra containing all open subsets of $X$. If $(\Omega, \mathcal{A})$ and $(\Phi, \Sigma)$ are two measurable spaces, then $\Omega \otimes \Phi$ denotes the smallest $\sigma$-algebra on $\Omega \times \Phi$ which contains all the sets $A \times B$, where $A \in \Omega, B \in \Phi$. Note that the Borel $\sigma$ algebra $B(X \times Y)$ contains $B(X) \otimes B(Y)$, in general. The map $T:(\Omega, \mathcal{A}) \rightarrow 2^{X}$ is said to have a measurable graph if Graph $T=\{(\omega, y) \in \Omega \times X: y \in T(\omega)\} \in \mathcal{A} \otimes B(X)$ (see [17]).

Tarafdar, Watson and Yuan [17] have proved the following. 
Theorem C: ([17, Theorem 7]). Let $(\Omega, \Sigma, \mu)$ be a measurable space with a positive measure $\mu$ (e.g. $\mu$ is an outer measure) and $X$ a nonempty Polish set in a topological space E. Suppose $F: \Omega \times X \rightarrow 2^{E}$ is such that for every $\omega \in \Omega$, the set $\{x \in X$ : $x \in F(\omega, x)\} \neq \emptyset$ and Graph $F \in \Sigma \otimes B(X \times E)$. Then there exists a single-valued measurable mapping $f: \Omega \rightarrow X$ such that $f(\omega) \in F(\omega, f(\omega))$ for almost every $\omega \in \Omega$.

The existence of random fixed point in almost sense of a nonexpansive operator is established in the following theorem which generalizes Theorem 3.1 to the family $\mathcal{F}$. Here incidentally, we obtain the conclusion of Theorem 2.4 [3] for the sets which are not necessarily starshaped in a locally bounded topological vector space.

Theorem 3.10: Let $(\Omega, \mathcal{A}, \mu)$ be any measure space with positive measure $\mu$ (e.g., $\mu$ is an outer measure) and $S$ a nonempty compact subset of $X$. Suppose that $\mathcal{F}$ is a family of contractive jointly continuous functions associated with $S$ and $f: \Omega \times S \rightarrow S$ a nonexpansive random operator. Then $f$ has a random fixed point in almost sense.

Proof: Clearly, $S$ is separable and metrizable under the complete metric $d_{p}$. Hence $S$ is a Polish set. It is well known that each jointly measurable single-valued mapping has a measurable graph and each random continuous single-valued mapping defined in a product domain is jointly measurable (cf. [17, p. 309]). Moreover, $\{x \in S: F(\omega, x)=x\}$ is nonempty by Theorem A. The result now follows by the single-valued version of Theorem C.

Theorem 3.11: Let $(\Omega, \mathcal{A}, \mu), \mathcal{F}$ and $f$ be as in Theorem 3.10. If $S$ is a separable weakly compact subset of $X$ and $X$ has separating dual $X^{*}$, then $f$ has a random fixed point in almost sense.

Proof: Similar to the proof of Theorem 3.10 with the exception that we apply Theorem B instead of Theorem A to show that $f(\omega, \cdot)$ has a fixed point for each $\omega \in \Omega$.

In the absence of the family $\mathcal{F}$ or $\mathcal{H}$, random fixed points for nonexpansive random operators may not exist as is clear from the following.

Example 3.12: (cf. [10, Example 3.5]). Let $S=\{0,1\}$ and $\left(\Omega, 2^{\Omega}\right)$ be a measurable space. Define $T: \Omega \times S \rightarrow S$ by

$$
T(\omega, 0)=1 \text { and } T(\omega, 1)=0 .
$$

Then $T$ is a nonexpansive random operator with no random fixed point because if there is any measurable function $\xi: \Omega \rightarrow S$ such that $\xi(\omega)=T(\Omega, \xi(\omega)), \omega \in \Omega$, then either

(i) $\xi(\omega)=0, \omega \in \Omega$,

(ii) $\xi(\omega)=1, \omega \in \Omega$,

or

(iii) $\xi(\omega)=0$ for some $\omega \in \Omega$

and $\xi(\omega)=1$ for some $\omega \in \Omega$ and obviously equation $(* *)$ does not hold in all the three cases.

Remarks 3.13: $(i)$ For the corresponding deterministic results of Theorems 3.1-3.2 and Theorems 3.5-3.6, we refer to [11].

(ii) Theorems 3.1-3.2 and Theorems 3.5-3.6 hold in the setup of the Fréchet space (see [1] for the corresponding deterministic results).

(iii) Theorem 3.5 removes the condition of convexity and the fixed point property of $S$ and the strict convexity of $X$ required in Theorem 1 of $\mathrm{Xu}$ [19].

(iv) Example 3.12 contradicts Theorem 2.2 and hence Theorem 3.2 of [20]. 


\section{Acknowledgements:}

The authors, A.R. Khan and A.B. Thaheem gratefully acknowledge the support provided by the King Fahd University of Petroleum and Minerals during this research. The authors are also grateful to the referee for useful suggestions leading to the improvement of the paper.

\section{References}

[1] Anderson, D.E., Nelson, J.L., and Singh, K.L., Fixed points for single and multivalued mappings in locally convex spaces, Math. Japon. 31 (1986), 665-672.

[2] Beg, I. and Shahzad, N., Random approximations and random fixed point theorems, $J$. Appl. Math. Stoch. Anal. 7 (1994), 145-150.

[3] Beg, I. and Shahzad, N., Random fixed points and approximations in random convex metric spaces, J. Appl. Math. Stoch. Anal. 6 (1993), 237-246.

[4] Brosowski, B., Fix Punktsätze in der Approximationstheorie, Mathematica (cluj), 11 (1969), 195-220.

[5] Bharucha-Reid, A.T., Fixed point theorems in probabilistic analysis, Bull. Amer. Math. Soc. 82 (1976), 641-657.

[6] Dotson, W.G., On fixed points of nonexpansive mappings in nonconvex sets, Proc. Amer. Math. Soc. 38 (1973), 155-156.

[7] Habinaik, L., Fixed point theorems and invariant approximations, J. Approx. Theory 56 (1989), 241-244.

[8] Himmelberg, C.J., Measurable relations, Fund. Math. 87 (1975), 53-72.

[9] Joshi, M.C. and Bose, R.K., Some Topics in Nonlinear Analysis, Wiley Eastern Ltd., New Delhi 1985.

[10] Khan, A.R. and Hussain, N., Random fixed points for *-nonexpansive random operators, J. Appl. Math. Stoch. Anal. 14 (2001), 341-349.

[11] Khan, A.R., Hussain, N., and Thaheem, A.B., Applications of fixed point theorems to invariant approximation, Approx. Theory and Appl. 16 (2000), 48-55.

[12] Khan, L.A. and Khan, A.R., An extension of Brosowski-Meinardus theorem on invariant approximations, Approx. Theory and Appl. 11 (1995), 1-5.

[13] Lin, T.C., Random approximations and random fixed point theorems for non-self maps, Proc. Amer. Math. Soc. 103:4 (1988), 1129-1135.

[14] Meinardus, G., Invarianze bei linearen approximationen, Arch. Rat. Mech. Anal. 14 (1963), 301-303.

[15] Mohebi, H., On quasi-Chebyshev subspaces of Banach spaces, J. Approx. Theory 107 (2000), 87-95.

[16] Subrahmanyam, P.V., An application of a fixed point theorem to best approximation, $J$. Approx. Theory 20 (1977), 165-172.

[17] Tarafdar, E., Watson, P., and Yuan, X.Z., The measurability of Caratheodory set-valued mappings and random fixed point theorems, Acta Math. Hungar. 74 (1997), 309-319.

[18] Veeramani, P., On some fixed point theorems on uniformly convex Banach spaces, $J$. Math. Anal. Appl. 167 (1992), 160-166.

[19] Xu, H.K., Some random fixed point theorems for condensing and nonexpansive operators, Proc. Amer. Math. Soc. 110 (1990), 495-500.

[20] Yi, H.W. and Zhao, Y.C., Fixed point theorems for weakly inward multivalued mappings and their randomization, J. Math. Anal. Appl. 183 (1994), 613-619. 


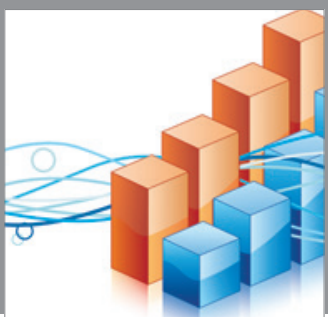

Advances in

Operations Research

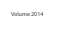

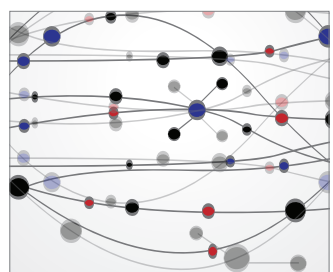

\section{The Scientific} World Journal
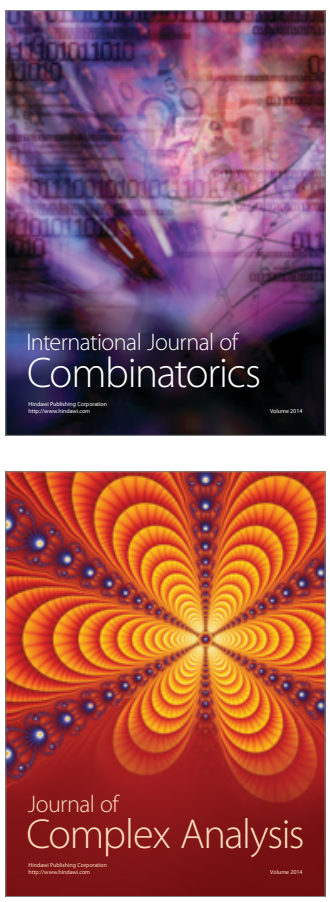

International Journal of

Mathematics and

Mathematical

Sciences
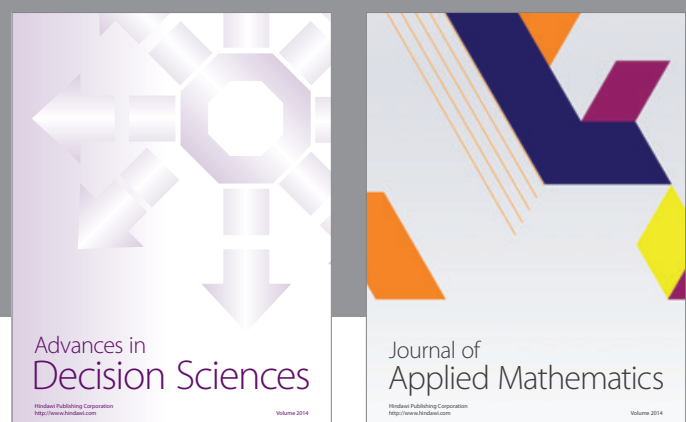

Journal of

Applied Mathematics
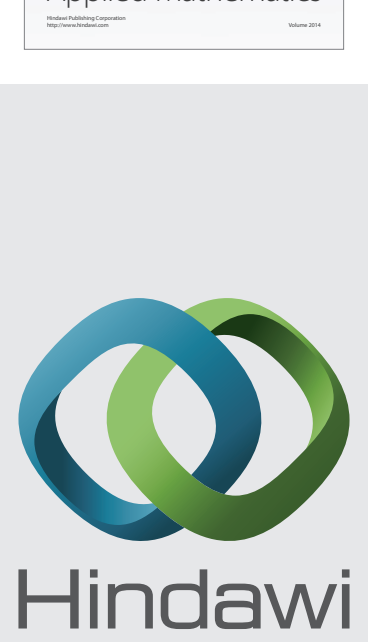

Submit your manuscripts at http://www.hindawi.com
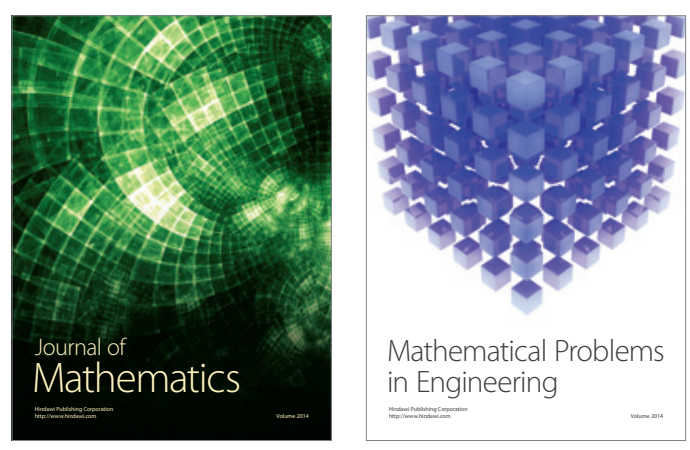

Mathematical Problems in Engineering
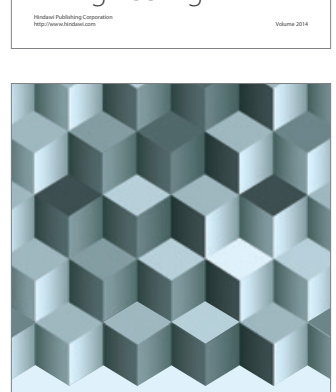

Journal of

Function Spaces
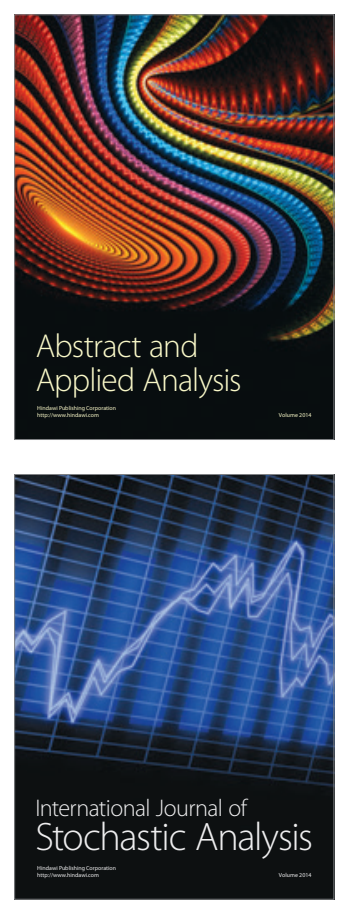

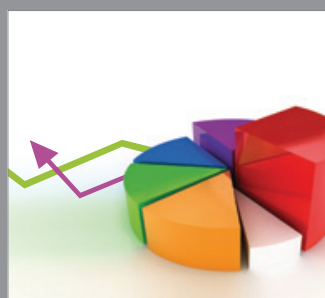

ournal of

Probability and Statistics

Promensencen
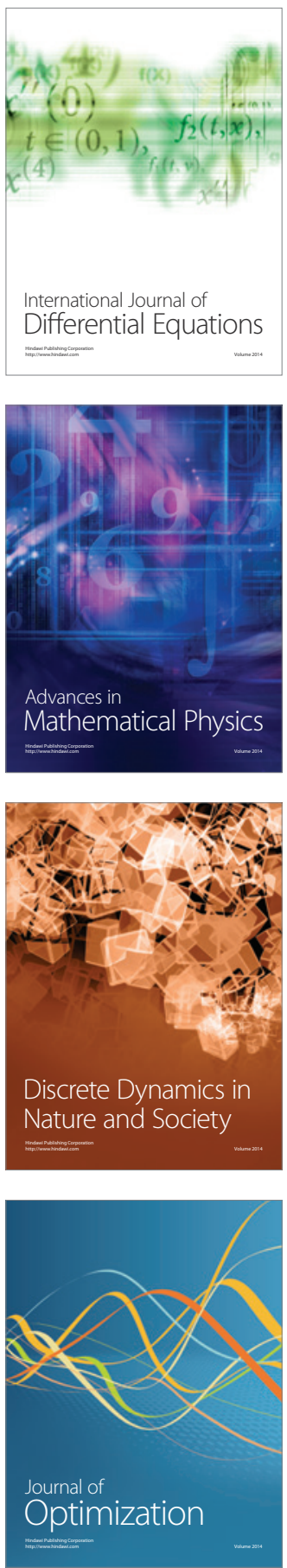